\title{
DIGITALCOMMONS
}

@WAYNESTATE —

Wayne State University

Mathematics Research Reports

Mathematics

9-1-2001

\section{Ultraconvergence of ZZ Patch Recovery at Mesh Symmetry Points}

Zhimin Zhang

Wayne State University, zhimin.zhang@wayne.edu

Runchang Lin

Wayne State University

\section{Recommended Citation}

Zhang, Zhimin and Lin, Runchang, "Ultraconvergence of ZZ Patch Recovery at Mesh Symmetry Points" (2001). Mathematics Research Reports. Paper 1.

http://digitalcommons.wayne.edu/math_reports/1

This Technical Report is brought to you for free and open access by the Mathematics at DigitalCommons@WayneState. It has been accepted for inclusion in Mathematics Research Reports by an authorized administrator of DigitalCommons@WayneState. 


\title{
ULTRACONVERGENCE OF ZZ PATCH RECOVERY AT MESH SYMMETRY POINTS
}

\author{
ZHIMIN ZHANG and RUNCHANG LIN
}

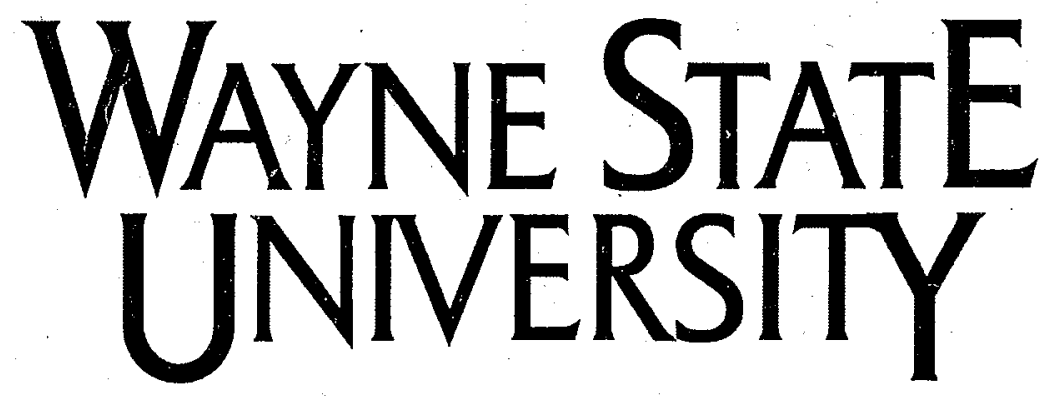

Detroit, MI 48202

Department of Mathematics

Research Report

\section{Series}

\#9

This research was partly supported by the National Science Foundation. 


\title{
Ultraconvergence of ZZ Patch Recovery at Mesh Symmetry Points
}

\author{
Zhimin Zhang*and Runchang Lin \\ Department of Mathematics, Wayne State University
}

\begin{abstract}
Ultraconvergence property of the Zienkiewicz-Zhu gradient patch recovery technique based on local discrete least squares fitting is established for a large class of even-order finite elements. The result is valid at all rectangular mesh symmetry points. Different smoothing strategies are discussed. Superconvergence recovery for the Q8 element is proved and ultraconvergence numerical examples are demonstrated.
\end{abstract}

Key Words. finite element method, ZZ-patch recovery, superconvergence, ultraconvergence

AMS Subject Classification. 65N30, 65N15, 65N12, 65D10, 74S05, 41A10, 41A25

\section{Introduction.}

Since the first appearance of the Zienkiewicz-Zhu patch recovery technique [15], a decade has been passed. Regardless of its great success in practice, the theoretical foundation behind this remarkable recovery technique has not yet been fully developed. There have been some limited theoretical results since the mid 90's. The reader is referred to two recent books by Ainsworth-Oden [1, Chapter 4] and by Babuška-Strouboulis. [4, Chapters 4,5] for discussion and references.

One of the fascinating features of $\mathrm{ZZ}$ patch recovery is its ultraconvergence property for quadratic elements which includes T6 (six-node triangular element), Q8 (eight-node serendipity element), and Q9 (nine-node tensor-product element). The term "ultraconvergence" indicates that the convergence rate is two orders higher than the optimal global rate. In an earlier work, the first author proved ultraconvergence for even-order elements at the vertices under locally uniform rectangular mesh [13].

The current work intends to view $\mathrm{ZZ}$ patch recovery from a different angle and to provide more insights on the mathematical reasoning behind the method. Our results can be divided into three parts. First, we investigate all possible smoothing strategies under the least squares fitting. This is done by concentrating on elements Q8 and Q9. In particular, we shall discuss the smoothing by quadratic polynomials (six terms), bi-quadratic polynomials (nine terms), as well as eight-term serendipity polynomials. We would like to remind the reader that only eight-term polynomial smoothing was numerically tested in the original work of Zienkiewicz-Zhu [15].

Secondly, we prove ultraconvergence of the recovered gradient for a large class of evenorder rectangular elements at all mesh symmetry points, which include vertices, edge cen-

*This research was partially supported by the National Science Foundation grants DMS-0074301, DMS0079743 , and INT-0196139. 
ters, and element centers. We would like to indicate that the ultraconvergence result at the element center was not in the original work of Zienkiewicz-Zhu [15], and hence it is a new result even numerically.

Thirdly, we establish superconvergence of the recovered gradient for the Q8 element at all mesh symmetry points and for all possible smoothing strategies. Furthermore, we provide some justification of ultraconvergence for Q8 that is supported by numerical evidence. Note that Q8 is the lowest even-order serendipity element.

\section{The ZZ Patch Recovery}

Consider an element patch which contains four rectangles that share a common vertex (assembly point). Assume that the four rectangles are uniform. We may further simplify to a square mesh. Then we can map the patch to the reference square $\hat{K}=[-1,1]^{2}$ by an affine mapping.

We discuss main features of the ZZ patch recovery by $\mathrm{Q} 8$ and $\mathrm{Q} 9$ elements. The ZZ patch recovery uses the sixteen Gaussian points (four from each element)

$$
\left( \pm \frac{1}{2} \pm \frac{1}{2 \sqrt{3}}, \pm \frac{1}{2} \pm \frac{1}{2 \sqrt{3}}\right) \text {, }
$$

denote as $G_{j}=\left(\xi_{j}, \eta_{j}\right), j=1,2, \ldots, 16$, as sampling points, where gradient values $\nabla u^{h}\left(g_{j}\right)$ of the finite element solutiontare calculated. Here $g_{j}$ is the Gaussian point in the original patch associated with $G_{j}$. A polynomial of degree $p=2$ is going to be constructed by a least-squares procedure. Along this line, there are three different strategies:

$$
\begin{aligned}
& p_{2}(\xi, \eta)=\left(1, \xi, \eta, \xi^{2}, \xi \eta, \eta^{2}\right)\left(a_{1}, a_{2}, \ldots, a_{6}\right)^{T} \\
& \bar{q}_{2}(\xi, \eta)=\left(1, \xi, \eta, \xi^{2}, \xi \eta, \eta^{2}, \xi^{2} \eta, \xi \eta^{2}\right)\left(a_{1}, a_{2}, \ldots, a_{8}\right)^{T} \\
& q_{2}(\xi, \eta)=\left(1, \xi, \eta, \xi^{2}, \xi \eta, \eta^{2}, \xi^{2} \eta, \xi \eta^{2}, \xi^{2} \eta^{2}\right)\left(a_{1}, a_{2}, \ldots, a_{9}\right)^{T}
\end{aligned}
$$

We describe the procedure using $p_{2}(\xi, \eta)$ whose coefficients $\vec{a}=\left(a_{1}, a_{2}, \ldots, a_{6}\right)^{T}$ will be determined by fitting data $\vec{\sigma}^{h}=\left(\sigma_{1}^{h}, \sigma_{2}^{h}, \ldots, \sigma_{16}^{h}\right)^{T}$ at those 16 Gaussian points in a leastsquares manner. Here $\sigma_{j}^{h}$ can be either one of the components of $\nabla u^{h}\left(g_{j}\right)$. This procedure results in a linear system

$$
B^{T} B \vec{a}=B^{T} \vec{\sigma}^{h}
$$

where

$$
B=\left(\begin{array}{cccccc}
1 & \xi_{1} & \eta_{1} & \xi_{1}^{2} & \xi_{1} \eta_{1} & \eta_{1}^{2} \\
1 & \xi_{2} & \eta_{2} & \xi_{2}^{2} & \xi_{2} \eta_{2} & \eta_{2}^{2} \\
\vdots & \vdots & \vdots & \vdots & \vdots & \vdots \\
1 & \xi_{16} & \eta_{16} & \xi_{16}^{2} & \xi_{16} \eta_{16} & \eta_{16}^{2}
\end{array}\right)
$$

Solve for $\vec{a}$, we obtain $p_{2}(\xi, \eta)$. Then $p_{2}(0,0)$ will be assigned as (recovered derivative value at the patch center (assembly point), $p_{2}(0, \pm 1 / 2)$ and $p_{2}( \pm 1 / 2,0)$ will be used to obtain recovered derivative values at four interior edge centers, and $p_{2}( \pm 1 / 2, \pm 1 / 2)$ will be used to obtain recovered derivative values at four element centers. In fact, the recovered derivative 
value at an edge center is the average from two overlapping patches (Figures 2, 3) and the recovered derivative value at an element center is the average from four overlapping patches (Figure 4). In this way, we can reconstruct derivative values at nine nodes on each element. By interpolation using original Q9 or Q8 basis functions, we then recover a piecewise continuous gradient field, which denoted as $G_{h} u^{h}$. Let $z$ be either a vertex, an edge center, or an element center, we can write

$$
G_{h} u^{h}(z)=\sum_{j=1}^{n} b_{j}(z) \nabla u^{h}\left(g_{j}\right), \quad \sum_{j=1}^{n} b_{j}(z)=1,
$$

where $b_{j}(z)$ 's are weights obtained from the above least-squares fitting procedure. Note that $n=16$ if $z$ is a vertex when only one element patch is involved (Figure 1), $n=24$ if $z$ is an edge center when two overlapping patches are involved (Figure 2), and $n=36$ if $z$ is an element center when four overlapping patches are involved (Figure 3).

Since each $\nabla u^{h}\left(g_{j}\right)$ can be expressed by the nodal values of the finite element solution, we have another expression:

$$
G_{h} u^{h}(z)=\frac{1}{h} \sum_{j=1}^{m} \vec{c}_{j}(z) u^{h}\left(z_{j}\right), \quad \sum_{j=1}^{m} \vec{c}_{j}(z)=\overrightarrow{0},
$$

with $m=25$ for Q9 and $m=21$ for Q8 if $z$ is a vertex, $m=35$ for Q9 and $m=29$ for Q8 if $z$ is an edge center, and $m=49$ for Q9 and $m=40$ for Q8 if $z$ is an element center.

The recovery operator $G_{h}$ is then completely based on the weights $\vec{c}_{j}$ 's. Therefore, we need to calculate those weights in order to obtain $G_{h}$. Since we have two different elements Q8 and Q9, and three smoothing (or recovery) strategies (2.1)-(2.3), there are totally six .cases, namely, Q8- $p_{2}, \mathrm{Q} 8-\bar{q}_{2}, \mathrm{Q} 8-q_{2}, \mathrm{Q} 9-p_{2}, \mathrm{Q} 9-\bar{q}_{2}$, and Q9- $q_{2}$. In each case, there are four sets of data for $\vec{c}_{j}$ 's at: vertex, horizontal edge center, vertical edge center, and element center.

Remark 2.1. Even for the Q8 element, the recovered gradient $G_{h} u^{h}$ can have a Q9 interpolation, whose values at vertices, edge centers, and element centers are uniquely determined by either (2.5) or (2.6).

Remark 2.2. In the original paper of Zienkiewicz-Zhu, only the case Q8- $\bar{q}_{2}$ was numerically tested and the recovery at the element center was not discussed.

With help of symbolic tools in Maple, we have calculated the first components ( $x$ derivative) of all twenty-four sets of weights $\vec{c}_{j}(z)$ 's. They are all different. However, we only provide data for the most economical case Q8- $p_{2}$ in Figures 1-4.

Note that all weights are distributed anti-symmetrically with respect to $z$ and the vertical lines passing through $z$ for the recovered $x$-derivative. Therefore, the recovered gradient is actually a finite difference scheme:

$$
G_{h} u^{h}(z)=\frac{1}{h} \sum_{j=1}^{N} \vec{c}_{j}(z)\left[u^{h}\left(z+h\left(\begin{array}{c}
\alpha_{j} \\
\beta_{j}
\end{array}\right)\right)-u^{h}\left(z-h\left(\begin{array}{c}
\alpha_{j} \\
\beta_{j}
\end{array}\right)\right)\right],
$$

where $N \leq[m / 2]$ and $\left(\alpha_{j}, \beta_{j}\right)$ 's are the $(\xi, \eta)$ coordinates of nodes in the reference square associated with the weight $\vec{c}_{j}(z)$. 
(twith foets 4$)$

For example, data in Figure 16 nodal values on four elements surrounding a vertex.

$$
\begin{aligned}
G_{h} u(z)= & \frac{1}{48 h}\left\{16\left[u\left(z+h\left(\begin{array}{c}
1 / 2 \\
0
\end{array}\right)\right)-u\left(z-h\left(\begin{array}{c}
1 / 2 \\
0
\end{array}\right)\right)\right]+2\left[u\left(z+h\left(\begin{array}{l}
1 \\
0
\end{array}\right)\right)+u\left(z-h\left(\begin{array}{c}
1 \\
0
\end{array}\right)\right)\right\}\right. \\
& +8\left[u\left(z+h\left(\begin{array}{c}
1 / 2 \\
1
\end{array}\right)\right)-u\left(z-h\left(\begin{array}{c}
1 / 2 \\
1
\end{array}\right)\right)+u\left(z+h\left(\begin{array}{c}
1 / 2 \\
-1
\end{array}\right)\right)-u\left(z-h\left(\begin{array}{c}
1 / 2 \\
-1
\end{array}\right)\right)\right] \\
& +4\left[u\left(z+h\left(\begin{array}{c}
1 \\
1 / 2
\end{array}\right)\right)-u\left(z-h\left(\begin{array}{c}
1 \\
1 / 2
\end{array}\right)\right)+u\left(z+h\left(\begin{array}{c}
1 \\
-1 / 2
\end{array}\right)\right)-u\left(z-h\left(\begin{array}{c}
1 \\
-1 / 2
\end{array}\right)\right)\right] \\
& \left.+5\left[u\left(z+h\left(\begin{array}{c}
-1 \\
1
\end{array}\right)\right)-u\left(z-h\left(\begin{array}{c}
-1 \\
1
\end{array}\right)\right)-u\left(z+h\left(\begin{array}{l}
1 \\
1
\end{array}\right)\right)+u\left(z-h\left(\begin{array}{l}
1 \\
1
\end{array}\right)\right)\right]\right\} .
\end{aligned}
$$

Data in Figures 2 represent a finite difference scheme which involves 26 nodal values on six elements surrounding an horizontal edge center. Each entry of three columns in the middle has two numbers: the left one comes from evaluating $p_{2}^{\text {left }}(1 / 2,0)$ from the left four elements and the right one comes from evaluating $p_{2}^{\text {right }}(-1 / 2,0)$ from the right four elements.

Similarly, data in Figures 3 represent a finite difference scheme which involves 22 nodal values on six elements surrounding a vertical edge center. Again we show contribution from two overlapping patches. Finally, data in Figures 4 represent a finite difference scheme which involves 36 nodal values on nine elements surrounding an element center. This time, contribution from four overlapping patches are demonstrated.

Note that entries on the central vertical lines are all zero due to cancellation. The scheme for $y$-derivative can be obtained by rotating Figures 1-4 90 degrees.

We denote $\omega_{z}$, a set of elements surrounding $z$ such that $\omega_{z}$ contains: a) four elements if $z$ is a vertex, b) six elements if $z$ is an edge center, and c) nine elements if $z$ is an element center. See Figures 1-4.

Theorem 2.1. Let $u \in W_{\infty}^{5}\left(\omega_{z}\right)$, where $z$ is either a vertex, an edge center, or an element center. Then the recovery operator $G_{h}$ from either Q8 or Q9 element with any one of the smoothing (2.1)-(2.3) satisfies

$$
\left|G_{h} u(z)-\nabla u(z)\right| \leq C h^{4}|u|_{W_{\infty}^{5}\left(\omega_{z}\right)} .
$$

Proof: By the Taylor expansion, we have

$$
\begin{aligned}
& u\left(z+h\left(\begin{array}{c}
\alpha \\
\beta
\end{array}\right)\right)-u\left(z-h\left(\begin{array}{c}
\alpha \\
\beta
\end{array}\right)\right) \\
= & 2 h\left(\alpha \frac{\partial}{\partial x}+\beta \frac{\partial}{\partial y}\right) u(z)+\frac{h^{3}}{3}\left(\alpha \frac{\partial}{\partial x}+\beta \frac{\partial}{\partial y}\right)^{3} u(z)+R_{h}(u),
\end{aligned}
$$

where

$$
\left|R_{h}(u)\right| \leq C h^{5}|u|_{W_{\infty}^{5}\left(\omega_{z}\right)} .
$$

It is straightforward to verify that for all twenty-four cases,

$$
2 \sum_{j=1}^{N} \vec{c}_{j}(z) \alpha_{j}=1, \quad \sum_{j=1}^{N} \vec{c}_{j}(z) \beta_{j}=0
$$




$$
\begin{aligned}
& \sum_{j=1}^{N} \vec{c}_{j}(z) \alpha_{j}^{3}=0, \quad \sum_{j=1}^{N} \vec{c}_{j}(z) \alpha_{j}^{2} \beta_{j}=0, \\
& \sum_{j=1}^{N} \vec{c}_{j}(z) \alpha_{j} \beta_{j}^{2}=0, \quad \sum_{j=1}^{N} \vec{c}_{j}(z) \beta_{j}^{3}=0 .
\end{aligned}
$$

Apply the Taylor expansion (2.9) to the right hand side of (2.7), simplify the result by (2.10)-(2.12), and we obtain

$$
G_{h} u(z)=\nabla u(z)+\frac{1}{h} R_{h}(u)
$$

the conclusion follows.

In other words, the recovery operator $G_{h}$ preserves polynomials of degree up to 4 at vertices, edge centers, and element centers when uniform rectangular elements are used locally. Indeed, this property is essential for a successful ultraconvergence recovery of the operator.

Remark 2.3. Both expression (2.5) and (2.6) are valid for $v \in V^{h}$, the finite element space of either Q8 or Q9. However, only expression (2.6) is valid for $u \notin V^{h}$, since $\nabla u$ cannot be expressed by its nodal values the same way as $\nabla u^{h}$ does in general.

Remark 2.4. Comparing with a simple fourth-order finite difference scheme (along one line)

$$
D_{h} u(0)=\frac{1}{6 h}[u(-h)-8 v(-h / 2)+8(h / 2)-v(h)], \quad u_{x}(0)-D_{h} u(0)=O\left(h^{4}\right),
$$

the advantage of those 24 schemes obtained by the ZZ patch recovery is their numerical stability under mesh distortion.

The verification of (2.10)-(2.12) can be done symbolically or numerically by computer. The following Matlab code verifies the first component of $(2.10)-(2.12)$ for the Q8- $p_{2}$ case when $z$ is the patch center (Figure 1). By symmetry, only $8 c_{j}$ 's and the associated $(\xi, \eta)$ coordinates are needed.

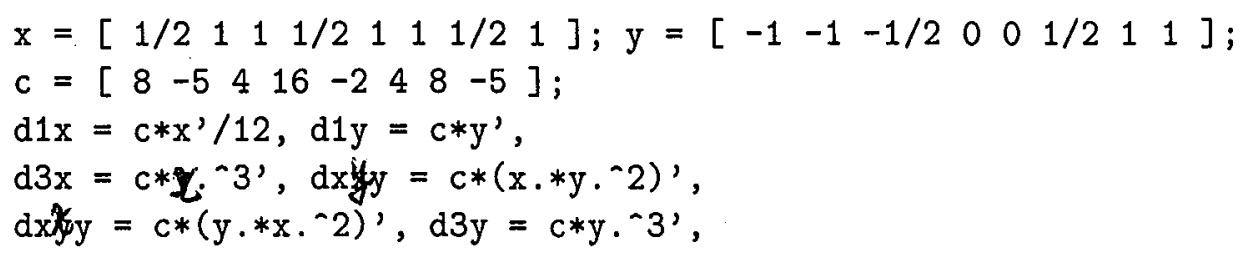

\section{Relationship of Some Popular Interpolations}

In this section, we consider three different quadratic interpolations on a rectangular element $K$ with vertices $z_{1}^{K}, z_{2}^{K}, z_{3}^{K}, z_{4}^{K}$, and edge centers $z_{5}^{K}, z_{6}^{K}, z_{7}^{K}, z_{8}^{K}$ (Figure 5). Without confusion, we suppress the index $K$ in order to simplify the notation. 
On the reference element $\hat{K}$, we can express them as

$$
u^{I}=\sum_{j=1}^{8} u_{j} N_{j}, \quad \bar{u}^{I}=\sum_{j=1}^{4} u_{j} N_{j}+\sum_{j=5}^{8} \bar{u}_{j} N_{j}, \quad \tilde{u}^{I}=\bar{u}^{I}+\tilde{u}_{9} N_{9}
$$

where $u_{j}=u\left(z_{j}\right), j=1,2, \ldots, 8$, and $N_{j}, j=1,2, \ldots, 9$, are conventional shape functions for quadrilaterals [10, p.101]:

$$
\begin{aligned}
N_{1} & =\frac{1}{4} \xi \eta(1-\xi)(1-\eta), & N_{2} & =\frac{1}{4} \xi \eta(1+\xi)(\eta-1), \\
N_{3} & =\frac{1}{4} \xi \eta(1+\xi)(1+\eta), & N_{4} & =\frac{1}{4} \xi \eta(\xi-1)(1+\eta), \\
N_{5} & =\frac{1}{2} \eta\left(1-\xi^{2}\right)(\eta-1), & N_{6} & =\frac{1}{2} \xi(1+\xi)\left(1-\eta^{2}\right), \\
N_{7} & =\frac{1}{2} \eta\left(1-\xi^{2}\right)(1+\eta), & N_{8} & =\frac{1}{2} \xi(\xi-1)\left(1-\eta^{2}\right), \\
& N_{9}=\left(1-\xi^{2}\right)\left(1-\eta^{2}\right) . & &
\end{aligned}
$$

We see that all three interpolate $u$ at the four vertices, and $u^{I}$ is the standard 8-node Lagrange interpolation. Coefficient $\bar{u}_{5}$ is defined by

$$
\int_{l_{1}} \partial_{s}\left(u-\bar{u}^{I}\right) \partial_{s} v d s, \quad \forall v \in V^{h}
$$

where $l_{1}$ is the edge linking $z_{1}$ and $z_{2}$, and $\partial_{s}$ is the tangential direction along $l_{1}$. The other three parameters, $\bar{u}_{6}, \bar{u}_{7}$, and $\bar{u}_{8}$ are defined in the same way.

Finally $\tilde{u}_{9}$ is defined by

$$
\int_{K} \nabla\left(u-\tilde{u}^{I}\right) \nabla v=0
$$

where $v$ is the interior shape function ( $N_{9}$ on the reference element).

Remark 3.1. Projection type interpolations such as $\bar{u}^{I}$ and $\tilde{u}^{I}$ are more "closer" to the finite element solution than the traditional Lagrange interpolation $u^{I}$. Projections (3.1) and (3.2) are also used in practical finite element codes such as StressCheck.

Lemma 3.1. The coefficients $\bar{u}_{5}$ can be equivalently defined by

$$
\int_{l_{1}}\left(u-\vec{u}^{I}\right) d s=0
$$

Proof: Performing integration by parts, we have

$$
\int_{l_{1}} \partial_{s}\left(u-\bar{u}^{I}\right) \partial_{s} v d s=-\int_{l_{1}}\left(u-\bar{u}^{I}\right) \partial_{s}^{2} v d s
$$

since $\bar{u}^{I}$ equals $u$ at the two ends of $l_{1}$. Note that $\partial_{s}^{2} v$ is a constant along $l_{1}$ for $v \in V^{h}, \mathrm{Q} 8$ or Q9 finite element, therefore (3.1) is equivalent to (3.3). 
Theorem 3.2. Let $u \in W_{\infty}^{5}(K)$, then

$$
\begin{aligned}
& \bar{u}_{j}=u_{j}-\frac{h^{4}}{1920} \partial_{x}^{4} u\left(z_{j}\right)+R_{h}^{x}(u), \quad j=5,7 \\
& \bar{u}_{j}=u_{j}-\frac{h^{4}}{1920} \partial_{y}^{4} u\left(z_{j}\right)+R_{h}^{y}(u), \quad j=6,8
\end{aligned}
$$

where

$$
\left|R_{h}^{x}(u)\right| \leq C h^{5}\left|\partial_{x}^{5} u\right|_{L_{\infty}(K)}, \quad\left|R_{h}^{y}(u)\right| \leq C h^{5}\left|\partial_{y}^{5} u\right|_{L_{\infty}(K)}
$$

Proof: A direct calculation shows that

$$
\int_{l_{1}} u^{I} d s=\frac{h}{2} \int_{-1}^{1}\left[u_{1} N_{1}(\xi,-1)+u_{5} N_{5}(\xi,-1)+u_{2} N_{2}(\xi,-1)\right] d \xi=\frac{h}{6}\left(u_{1}+4 u_{5}+u_{2}\right) .
$$

This is the Simpson integration with the error estimate

$$
\int_{l_{1}} u d s=\frac{h}{6}\left(u_{1}+4 u_{5}+u_{2}\right)-\frac{h^{5}}{2880} \partial_{x}^{4} u\left(z_{5}\right)+\frac{2 h}{3} R_{h}^{x}(u)
$$

which can be obtained by modifying the proof in [3, p.257]. Similarly

$$
\int_{l_{1}} \bar{u}^{I} d s=\frac{h}{6}\left(u_{1}+4 \bar{u}_{5}+u_{2}\right)
$$

By (3.3), we have

$$
\frac{h}{6}\left(u_{1}+4 u_{5}+u_{2}\right)-\frac{h^{5}}{2880} \partial_{x}^{4} u\left(z_{5}\right)+\frac{2 h}{3} R_{h}^{x}(u)=\frac{h}{6}\left(u_{1}+4 \bar{u}_{5}+u_{2}\right) .
$$

Therefore,

$$
\bar{u}_{5}=u_{5}-\frac{h^{4}}{1920} \partial_{x}^{4} u\left(z_{5}\right)+R_{h}^{x}(u) .
$$

The same argument is valid for $\bar{u}_{6}, \bar{u}_{7}$, and $\vec{u}_{8}$.

Remark 3.2. Theorem 2 discloses the difference between the 8-node projection type interpolation and the 8-node Lagrange interpolation. The result implies that when the target function $u$ is a fourth degree polynomial without $x^{4}$ and $y^{4}$ terms, the two interpolations will be the same.

\section{Ultraconvergence Property of the Recovery}

In this section, we will prove a theorem for the recovery operator $G_{h}$ in a more general setting and thereby establish ultraconvergence at the mesh symmetry points for even-order finite element methods. Our general theory covers the Q9 element. However, further analysis is required for the Q8 element.

Definition 4.1. The projection type interpolation $u^{I}$ of a function $u$ onto a $C^{0}$ finite element space $V^{h}$ satisfies:

(a) $u^{I}\left(z_{i}\right)=u\left(z_{i}\right)$ at all vertices $z_{i}$. 
(b) For all edge mode (on an edge $l$ ) $v \in V^{h}$,

$$
\int_{l} \partial_{s}\left(u^{I}-u\right) \partial_{s} v d s=0
$$

where $\partial_{s}$ is the tangential derivative along $l$.

(c) For all interior modes (on an element $K$ ) $v \in V^{h}$,

$$
\int_{K} \nabla\left(u^{I}-u\right) \nabla v=0
$$

The reader is referred to [10] for details about edge modes and interior modes.

Definition 4.2. The intermediate family of type I with degree $r$ is a $C^{0}$ finite element with local space $P_{r+1}(\hat{K}) \backslash \operatorname{Span}\left\{\xi^{r+1}, \eta^{r+1}\right\}$. Here $\hat{K}$ is the reference element and $P_{r}$ is the space of complete polynomials of degree $\leq r$.

The intermediate family of type II with degree $r$ is a $C^{0}$ finite element with local space $P_{r+2}(\hat{K}) \backslash \operatorname{Span}\left\{\xi^{r+1}, \eta^{r+1}, \xi^{r+2}, \eta^{r+2}, \xi^{r+1} \eta, \xi \eta^{r+1}\right\}$.

Theorem 4.1. Assume that $u \in W_{\infty}^{2 k+3}\left(\omega_{z}\right)$, where $\omega_{z}$ is the set of rectangular elements involved by $G_{h}$ at a mesh symmetry point $z$ and $G_{h}$ is the gradient recovery operator obtained from the $\mathrm{ZZ}$ least squares patch recovery procedure with at least $P_{2 k}$ smoothing. Let $u^{I}$ be the projection type interpolation of $u$ in a $C^{0}$ finite element space that contains the intermediate family of type I with degree $2 k$, Then there exists a constant $C$ independent of $u, h$, and $z$, such that

$$
\left|\nabla u(z)-G_{h} u^{I}(z)\right| \leq C h^{2 k+2}|u|_{W_{\infty}^{2 k+3}\left(\omega_{z}\right)},
$$

Proof: let $u \in P_{2 k+2}\left(\omega_{z}\right)$ and $z=\left(x_{0}, y_{0}\right)$. Then $u$ can be decomposed into $u=p+q$ with $q \in P_{2 k+1}\left(\omega_{z}\right)$ and

$$
p(x, y)=\sum_{j=0}^{2 k+2} a_{j}\left(x-x_{0}\right)^{j}\left(y-y_{0}\right)^{2(k+1)-j} .
$$

Clearly $\nabla p(z)=\overrightarrow{0}$, and consequently $\nabla u(z)=\nabla q(z)$.

Consider $u^{I}=p^{I}+q^{I}$ and we express

$$
G_{h} q^{I}(z)=\sum_{j} b_{j}(z) \nabla q^{I}\left(g_{j}\right), \quad G_{h} p^{I}(z)=\frac{1}{h} \sum_{j} \vec{c}_{j}(z) p^{I}\left(z_{j}\right)
$$

where $g_{j}$ and $z_{j}$ are the Gaussian points and element nodal degrees of freedom on $\omega_{z}$, respectively. Note that $\vec{c}_{j}(z)$ are anti-symmetrically distributed and $p^{I}$ is an even function (as the interpolation of an even polynomial $p$ ) with respect to the mesh symmetry point $z$, therefore,

$$
G_{h} p^{I}(z)=\frac{1}{2 h} \sum_{j} \vec{c}_{j}(z)\left[p^{I}\left(z_{j}\right)-p^{I}\left(z-\left(z_{j}-z\right)\right)\right]=0 .
$$


On the other hand, $q^{I} \in P_{2 k+1}\left(\omega_{z}\right) \backslash \operatorname{Span}\left\{\xi^{2 k+1}, \eta^{2 k+1}\right\}$ is the projection type interpolation of $q \in P_{2 k+1}(\omega)$, then $\nabla q^{I}\left(g_{j}\right)=\nabla q\left(g_{j}\right)$ at all Gaussian points $g_{j}$ on $\omega_{z}$. Hence,

$$
G_{h} q^{I}(z)=\sum_{j} b_{j}(z) \nabla q^{I}\left(g_{j}\right)=\sum_{j} b_{j}(z) \nabla q\left(g_{j}\right)=\nabla q(z) .
$$

The last equality based on the fact that a least squares fitting, by a $P_{2 k}$ polynomial, of exact values must reproduce the original polynomial. Note that $\nabla q \in P_{2 k}\left(\omega_{z}\right)^{2}$.

Observe that $G_{h}$ is a linear operator, then from (4.1) and (4.2),

$$
G_{h} u^{I}(z)=G_{h} p^{I}(z)+G_{h} q^{I}(z)=\nabla q(z)=\nabla u(z) .
$$

Since (4.3) holds for all polynomials of degree $2 k+2$, the conclusion follows from the Bramble-Hilbert Lemma [7, Theorem 4.1.3] and a scaling argument. Since $b_{j}(z)$ 's and $\vec{c}_{j}(z)$ 's depend only on mesh patterns around $z$, not the particular location of $z$, hence, the norm of $G_{h}$ is independent of $z$.

Remark 4.1. Theorem 4.1 is valid for all three interpolations in Section 3 because of Theorem 3.2. Note that Q8 belongs to both serendipity family and intermediate family of type I with degree 2.

Theorem 4.2. Let $u^{h}$ be the finite element approximation of the Poisson equation in a $C^{0}$ finite element space that contains the intermediate family of type II with degree $2 k$, let $G_{h}$ be the gradient recovery operator obtained from the ZZ least-squares fitting procedure with at least $P_{2 k}$ smoothing, and let $z$ be a symmetry point in a locally uniform rectangular mesh (on $D$ ) such that $\omega_{z} \subset \subset D \subset \subset \Omega$. Assume that $u \in W_{\infty}^{2 k+3}(D) \cap H^{2}(\Omega)$. Then there exists a constant $C$ independent of $u, h$, and $z$, such that

$$
\left|\nabla u(z)-G_{h} u^{h}(z)\right| \leq C h^{2(k+1)}\left|\ln h\left\|\left.u\right|_{W_{\infty}^{2 k+3}(D)}+C\right\| u-u^{h} \|_{H^{-l}(\Omega)},\right.
$$

for a positive integer $l \leq(3) 2 k+1 . \quad 2 k-1$

Proof: We start from the expression

$$
\nabla u(z)-G_{h} u^{h}(z)=\nabla u(z)-G_{h} u^{I}(z)+G_{h}\left(u^{I}-u^{h}\right)(z),
$$

where $u^{I}$ is the projection type interpolation as in Theorem 4.1. Using the following error estimate obtained from the interior analysis [13, Theorem 3.3],

$$
\left|u^{I}-u^{h}\right|_{W_{\infty}^{1}\left(\omega_{z}\right)} \leq C h^{2(k+1)}|\ln h||u|_{W_{\infty}^{2 k+3}(D)}+C\left\|u-u^{h}\right\|_{H^{-l}(\Omega)},
$$

we obtain

$$
\begin{aligned}
& \left|G_{h}\left(u^{I}-u^{h}\right)(z)\right| \leq C \max _{j}\left|\nabla\left(u^{I}-u^{h}\right)\left(g_{j}\right)\right| \\
& \leq C h^{2(k+1)}\left|\ln h\left\|\left.u\right|_{W_{\infty}^{2 k+3}(D)}+C\right\| u-u^{h} \|_{H^{-l}(\Omega)} .\right.
\end{aligned}
$$

The constant $C=\sum_{j}\left|b_{j}(z)\right|$ is independent of $z$ since $b_{j}(z)$ 's depend only on mesh patterns around $z$, not the particular location of $z$. The conclusion then follows by applying Theorem 4.1 and (4.6) to (4.4). 
Remark 4.2. According to [5, p.184], the term $\ln h$ can be removed for higher order finite elements. The reason it appears here is due to a technical reason since we quote a result from [13] which bears the logarithm term.

Remark 4.3. In the proof of Theorem 4.2, only (4.5) needs the assumption for the Poisson equation. The theorem can be applied to other second-order elliptic equations as long as (4.5) is satisfied, i.e., the projection interpolation $u^{I}$ is "ultra-close" to the the finite element solution.

Remark 4.4. Theorem 4.2 implies ultraconvergence local recovery when the solution is globally smooth or pollution effect caused by solution singularity is properly controlled such that the negative norm

$$
\left\|u-u^{h}\right\|_{H^{-l}(\Omega)}=O\left(h^{2(k+1)}\right) .
$$

See [8] for the discussion about the negative norm bounds under different regularity assumption on the solutions.

Remark 4.5. Theorem 4.2 generalizes the result in [13] from vertices to all mesh symmetry points including edge centers and element centers. By the symmetry theory $[9,11]$, gradient, or its average, of the finite element solution is superconvergent at a local mesh symmetry point for odd order elements (linear, cubic, ...), and finite element solution itself is superconvergent at a local mesh symmetry point for even-order elements. Here we have proved that the recovered gradient from the ZZ-patch least squares fitting is two order superconvergent at all mesh symmetry points for even-order elements.

Theorem 4.2 includes the Q9 element as a special case when $k=1$. However, it does not include the Q8 element since Q8 does not contain the intermediate family of type II. The analysis for the Q8 element is more complicated. We only provide a proof in a special situation, uniform square partition, although it is possible to establish some local theory by interior analysis. For a fix constant $\rho>0$, we define

$$
\Omega_{\rho}=\{x \in \Omega, \operatorname{dist}(x, \text { Corner set of } \Omega) \geq \rho\} .
$$

We use $S^{h}$ to denote Q8 finite element space and use $B_{h}$ to represent all bubble functions in the Q9 finite element space $V^{h}$. Clearly, $V^{h}=S^{h} \cup B_{h}$. Further, notation $S_{0}^{h}(D)$ indicates a finite element subspace with support on $D$. According to our notation, each $\tilde{u}^{I} \in V_{0}^{h}(\Omega)$ can be decomposed into $\tilde{u}^{I}=\bar{u}^{I}+u_{b}^{I}$ with $\bar{u}^{I} \in S_{0}^{h}(\Omega)$ and $u_{b}^{I} \in B_{h}(\Omega)$.

We need the following Lemma from [6, Lemma 10.7.4].

Lemma 4.3. Assume $u \in W_{\infty}^{6}(\Omega) \cap H_{0}^{1}(\Omega)$, Let $\mathcal{T}_{h}$ be a uniform square partition of $\Omega$. Then for any $v \in V_{0}^{h}(\Omega)$,

$$
\left(\nabla\left(u-\tilde{u}^{I}\right), \nabla v\right)=-\frac{h^{4}}{45} \int_{\Omega}\left(\partial_{x}^{4} \partial_{y}^{2} u+\partial_{x}^{2} \partial_{y}^{4} u\right) v+R_{h}(u, v)
$$

where

$$
\left|R_{h}(u, v)\right| \leq C h^{6}\|u\|_{W_{\infty}^{6}(\Omega)}\|v\|_{W_{1}^{2}(\Omega)}^{\prime}, \quad\|v\|_{W_{1}^{2}(\Omega)}^{\prime}=\left(\sum_{K \in \mathcal{T}_{h}}\|v\|_{W_{1}^{2}(K)}^{2}\right)^{1 / 2} .
$$

Theorem 4.4. Assume $u \in C^{6+\epsilon}(\bar{\Omega}) \cap H_{0}^{1}(\Omega)$. Let $\mathcal{T}_{h}$ be a uniform square partition of $\Omega$ and let $u^{h} \in S_{0}^{h}(\Omega)$ be the Q8 finite element solution of the Poisson equation on $\Omega$. Then 
there exists $w \in C^{2+\epsilon}\left(\Omega_{\rho}\right) \cap H^{2}(\Omega) \cap H_{0}^{1}(\Omega)$ such that at a mesh symmetry center (vertex, edge center, or element center) $z$,

$$
\left(u^{h}-\bar{u}^{I}\right)(z)=\left(\nabla u_{b}^{I}, \nabla g_{z}^{h}\right)+h^{4} w(z)+r_{h}^{z}(u)
$$

where $g_{z}^{h} \in S_{0}^{h}(\Omega)$ is the Q8 discrete Green's function and

$$
\begin{gathered}
\left|r_{h}^{z}(u)\right| \leq C(u) h^{6}|\ln h| \\
\left|\left(\nabla u_{b}^{I}, \nabla g_{z}^{h}\right)\right| \leq C h^{4}|\ln h||u|_{W_{\infty}^{4}(\Omega)} .
\end{gathered}
$$

Proof: Define $w \in H_{0}^{1}(\Omega)$ such that

$$
\Delta w=\frac{1}{45} \Delta \partial_{x}^{2} \partial_{y}^{2} u
$$

By the regularity theory of the elliptic boundary value problem, $w \in H^{2}(\Omega) \cap C^{2+\epsilon}\left(\Omega_{\rho}\right)$.

Set $v=g_{z}^{h}$ in (4.7), use the decomposition $\tilde{u}^{I}=\bar{u}^{I}+u_{b}^{I}$, and we have

$$
\begin{aligned}
\left(u^{h}-\bar{u}^{I}\right)(z)-\left(\nabla u_{b}^{I}, \nabla g_{z}^{h}\right) & =-\frac{h^{4}}{45}\left(\Delta \partial_{x}^{2} \partial_{y}^{2} u, g_{z}^{h}\right)+R_{h}\left(u, g_{z}^{h}\right) \\
& =h^{4} w^{h}(z)+R_{h}\left(u, g_{z}^{h}\right)
\end{aligned}
$$

where $w^{h} \in V_{0}^{h}(\Omega)$ is the Q8 finite element solution of $w$ which satisfies

$$
\left\|w-w^{h}\right\|_{L_{\infty}\left(\Omega_{\rho}\right)} \leq C h^{2}\left(\|w\|_{C^{2+\epsilon}\left(\Omega_{\rho}\right)}+\|w\|_{H^{2}(\Omega)}\right) \text {. }
$$

Note that $\left\|g_{z}^{h}\right\|_{W_{1}^{2}(\Omega)}^{\prime} \leq C|\ln h|$ (see $[6]$ or [14]). We then obtain (4.9) from (4.12) with

$$
r_{h}^{z}(u)=h^{4}\left(w^{h}-w\right)(z)+R_{h}\left(u, g_{z}^{h}\right)
$$

satisfies (4.10) in light of (4.8) and (4.13). As for (4.11), we have

$$
\left|\left(\nabla u_{b}^{I}, \nabla g_{z}^{h}\right)\right|=\left|\left(u_{b}^{I}, \Delta g_{z}^{h}\right)\right| \leq\left\|u_{b}^{I}\right\|_{L_{\infty}(\Omega)}\left\|g_{z}^{h}\right\|_{W_{1}^{2}(\Omega)}^{\prime} \leq C h^{4}|\ln h \| u|_{W_{\infty}^{4}(\Omega)} .
$$

Note that $\left.u_{b}^{I}\right|_{K}$ is the coefficient of the fourth order derivative $\partial_{x}^{2} \partial_{y}^{2} u$ on $K$, and it is of the 4 th order by the approximation theory. Since $z$ is arbitrary, we then establish (4.11). $\square$.

Theorem 4.5. Under the same assumption as in Theorem 4.4,

$$
\left|\left(G_{h} u^{h}-\nabla u\right)(z)\right| \leq C h^{3}\|u\|_{W_{\infty}^{4}\left(\omega_{z}\right)}+\text { higher order terms, }
$$

where $G_{h}$ is the ZZ patch recovery operator from either one of the smoothing (2.1)-(2.3), and $z$ is a mesh symmetry center.

Proof: Based on Theorem 4.4, we express

$$
\begin{aligned}
\left(G_{h} u^{h}-\nabla u\right)(z) & =G_{h}\left(u^{h}-\bar{u}^{I}\right)(z)+\left(G_{h} \bar{u}^{I}-\nabla u\right)(z) \\
& =G_{h}\left(\nabla u_{b}^{I}, \nabla g_{z}^{h}\right)+h^{4} G_{h} w(z)+G_{h} r_{z}^{h}+\left(G_{h} \bar{u}^{I}-\nabla u\right)(z) .
\end{aligned}
$$


We estimate each term on the right hand side. For the first term, we have

$$
\left|G_{h}\left(\nabla u_{b}^{I}, \nabla g_{z}^{h}\right)\right| \leq C h^{-1}\left|\left(\nabla u_{b}^{I}, \nabla g_{z}^{h}\right)\right| \leq C h^{3}|\ln h||u|_{W_{\infty}^{4}(\Omega)},
$$

by (4.11). Here $C=\sum_{j}\left|\vec{c}_{j}(z)\right|$ is independent of $z$ since $\vec{c}_{j}(z)$ 's depend only on mesh patterns around $z$, not the particular location of $z$. From Theorem 4.1, we know

$$
\left|\left(G_{h} \bar{u}^{I}-\nabla u\right)(z)\right| \leq C h^{4}|u|_{W_{\infty}^{5}\left(\omega_{z}\right)} .
$$

As a finite difference operator, $G_{h} w(z)=\nabla w(z)+O\left(h^{2}\right)$, therefore,

$$
h^{4}\left|G_{h} w(z)\right| \leq C h^{4}\|u\|_{W_{\infty}^{5}\left(\omega_{z}\right)}+\text { higher order terms. }
$$

Clearly, $\left|G_{h} r_{z}^{h}\right| \leq C(u) h^{5}$ by (4.10). Recall (4.15)-(4:17), we establish the assertion.

Remark 4.6. The obstacle prevents us from proving ultraconvergence of the Q8 element is the term $G_{h}\left(\nabla u_{b}^{I}, \nabla g_{z}^{h}\right)$. We only provide a pessimistic estimate (4.15) without taking advantage of the possible cancellation from the symmetry. However, we do believe that this term should be of order $O\left(h^{4}\right)$ under certain regularity of $u$. This can be partially justified by the following argument.

Clearly, $\left(\nabla u_{b}^{I}, \nabla g_{z}^{h}\right)$ is a function of $z$ which involves some discrete values of $\partial_{x}^{2} \partial_{y}^{2} u$. Note that the discrete Green's function $g_{z}^{h}$ decays exponentially away from $z$, therefore, only those values of $\partial_{x}^{2} \partial_{y}^{2} u$ near $z$ have impact on the recovery. Recall the anti-symmetry distribution of $\vec{c}_{j}(z)$ of the recovery operator $G_{h}$, we see that $G_{h}\left(\nabla u_{b}^{I}, \nabla g_{z}^{h}\right)$ will result in finite difference scheme of $\left(\nabla u_{b}^{I}, \nabla g_{z}^{h}\right)$ plus a higher order term that comes from the decay of $g_{z}^{h}$. By the scaling argument, we then have

$$
G_{h}\left(\nabla u_{b}^{I}, \nabla g_{z}^{h}\right)=C h^{4} \nabla \partial_{x}^{2} \partial_{y}^{2} u(z)+\text { higher order term }
$$

We notice that $\left(\nabla u_{b}^{I}, \nabla g_{z}^{h}\right)=u_{b}^{I, h}(z)$ is the Q8 finite element approximation of the bubble function $u_{b}^{I}(z)$. We further note that

$$
G_{h} u_{b}^{I}(z)=\frac{1}{h} \sum_{j} \vec{c}_{j}(z) u_{b}^{I}\left(z_{j}\right)=0=\nabla u_{b}^{I}(z),
$$

since the bubble function $u_{b}^{I}\left(z_{j}\right)=0$ when $z_{j}$ is an element vertex or edge center. Therefore,

$$
\left(G_{h} u_{b}^{I, h}-\nabla u_{b}^{I}\right)(z)=G_{h}\left(\nabla u_{b}^{I}, \nabla g_{z}^{h}\right)=C h^{4} \nabla \partial_{x}^{2} \partial_{y}^{2} u(z)+\text { higher order term. }
$$

This implies that if $u$ is a fourth order polynomial, then the recovered gradient of the Q8 finite element approximation to the bubble term $u_{b}^{I}$ will converge at an order higher than four at a mesh symmetry point. Our numerical test confirmed this observation. See Section 5 Example 3 for details.

\section{Numerical Test}

Ultraconvergence at vertices and edge centers were observed numerically in the original work of Zienkiewicz and Zhu [15]. However, the ultraconvergence at the element center by 
averaging of four overlapping patches is a new result. In addition, we shall consider "true" edge centers, comparing with "pseudo" edge centers of the original work in [15, §3.2], where the convergence of the recovered derivatives at edge centers are examined by the errors at the nearest edge center to a fixed vertex.

In order to preserve the element center and edge center in a coarser mesh to a fine mesh, we need to refine the mesh 3 -by-3, instead of 2-by-2. We start from a 4-by-4 mesh and pick following points

$$
\left(\frac{1}{4}, \frac{1}{4}\right), \quad\left(\frac{3}{8}, \frac{1}{4}\right), \quad\left(\frac{1}{4}, \frac{3}{8}\right), \quad\left(\frac{3}{8}, \frac{3}{8}\right),
$$

on the unit square $[0,1]^{2}$ as our vertex, horizontal edge center, vertical edge center, and element center, respectively. With 3-by-3 refinement, the next two mesh levels will be 12-by-12 and 36-by-36.

Example 1. In order to exclude the boundary singularity, our first example is

$$
-\Delta u=2 \pi^{2} \sin \pi x \sin \pi y \quad \text { in } \Omega=[0,1]^{2}, \quad u=0 \quad \text { on } \partial \Omega .
$$

The exact solution is $u(x, y)=\sin \pi x \sin \pi y$.

Example 2. Our second example is a modification of a test case in [15], the Poisson equation with zero boundary condition on the unit square with the exact solution

$$
u(x, y)=x(1-x) y(1-y)(1+2 x+7 y+23 x y) .
$$

Note that in this case, the Q9 element is exact at all nodal points (vertices, edge centers, and element centers), although seven terms

$$
x^{3}, y^{3}, x^{3} y, x y^{3}, x^{3} y^{2}, x^{2} y^{3}, x^{3} y^{3}
$$

are not in the Q9 finite element space. The original problem in [15] is

$$
u(x, y)=x(1-x) y(1-y)(1+2 x+7 y) .
$$

Figures 6 and 7 plot the convergence rate for the above two test cases at those four points in (5.1). We observe a perfect fourth order convergence rate.

Example 3. Our third example is to numerically verify the point we have made in Remark 4.6. We use Q8 finite element to solve the Poisson equation

$$
-\Delta u=2 x(1-x)+2 y(1-y)
$$

on the unit square with zero boundary condition. The exact solution is $u=x(1-x) y(1-y)$. Now the exact solution $u$ is in the Q9 finite element space and hence can be decomposed into $u=\bar{u}^{I}+u_{b}^{I}$ where $\bar{u}^{I}$ is the projection type interpolation as defined in Section 3 and $u_{b}$ is the bubble function. Since $\bar{u}^{I}$ is in the Q8 finite element space, it can be exactly resolved. Therefore, $u-u^{h}=u_{b}^{I}-u_{b}^{I, h}$. Note that in this case $G_{h} u=\nabla u$ on the whole domain. Therefore,

$$
\left(G_{h} u^{h}-\nabla u\right)(z)=\left(G_{h} u_{b}^{I, h}-\nabla u_{b}^{I}\right)(z)
$$


By (4.19), it should converge at an order higher than four. Indeed, our numerical test indicates a convergence rate close to six at an interior mesh symmetry point $z$. See Figure 8.

Conclusion. Ultraconvergence of the $\mathrm{ZZ}$ patch recovery technique (with any smoothing that includes $P_{2 k}$ ) for even-order $(2 k)$ rectangular finite elements that contains the intermediate family of type II has been proved at mesh symmetry points. We have proved not only what was observed by Zienkiewicz-Zhu, but also generalized their recovery to the element center and to higher order elements. In practice, the idea can be used for arbitrary quadrilaterals.

Although the least-squares procedure can be applied to general quadrilaterals, the ultraconvergence and superconvergence properties will disappear with mesh distortion. This is especially serious for the Q8 element. work by Arnold, Boffi, and Falk [2], the authors have shown that under certain quadrilateral mesh, the Q8 element cannot maintain a full quadratic $\left(P_{2}\right)$ approximation in the physical plane. In this respect, the reader is referred to a recent work [12] about a modified Q8 element. Nevertheless, in most practical situations, $\mathrm{ZZ}$ patch recovery is still able to produce much improved gradients numerically. A reason for this phenomenon is its numerical stability which is partially evidenced from Figures 1-4, i.e., a scheme based on more spread-out data is less sensitive to mesh distortion.

\section{References}

[1] M. Ainsworth and J.T. Oden, A Posteriori Error Estimation in Finite Element Analysis, Wiley Interscience, New York, 2000.

[2] D.N. Arnold, D. Boff, and R.S. Falk, Approximation by quadrilateral finite elements, to appear in Math. Comp.

[3] K.E. Atkinson, An Introduction to Numerical Analysis, 2nd Ed., John Wiley \& Sons, New York, 1989.

[4] I. Babuška and T. Strouboulis, The Finite Element Method and its Reliability, Oxford University Press, London, 2001.

[5] S.C. Brenner and L.R. Scott, The Mathematical Theory of Finite Element Methods, Springer-Verlag, New York, 1994.

[6] C.M. Chen and Y.Q. Huang, High Accuracy Theory of Finite Element Methods. Hunan Science Press, Hunan, China, 1995 (in Chinese).

[7] P.G. Ciarlet, The finite element method for elliptic problems, North-Holland, Amsterdam, 1978.

[8] J.A. Nitsche and A.H. Schatz, Interior estimates for Ritz-Galerkin methods, Math. Comp. 28 (1974), 937-958. 
[9] A.H. Schatz, I.H. Sloan, and L.B. Wahlbin, Superconvergence in finite element methods and meshes that are locally symmetric with respect to a point, SIAM J. Numer. Anal. 33 (1996), 505-521

[10] B. Szabó and I. Babuška, Finite Element Analysis, John Wiley \& Sons, New York, 1991.

[11] L.B. Wahlbin, Superconvergence in Galerkin Finite Element Methods, Lecture Notes in Mathematics, Vol.1605, Springer, Berlin, 1995.

[12] J. Zhang and F. Kikuchi, Interpolation error estimates of a modified 8-node serendipity finite element, Numer. Math. 85 (2000), 503-524.

[13] Z. Zhang, Ultraconvergence of the patch recovery technique II, Math. Comp. 69 (2000), $141-158$.

[14] Q.D. Zhu and Q. Lin, Superconvergence Theory of the Finite Element Method, Hunan Science Press, China, 1989 (in Chinese).

[15] O.C. Zienkiewicz and J.Z. Zhu, The superconvergence patch recovery and a posteriori error estimates. Part 1: The recovery technique, Int. J. Numer. Methods Engrg. 33 (1992), 1331-1364.

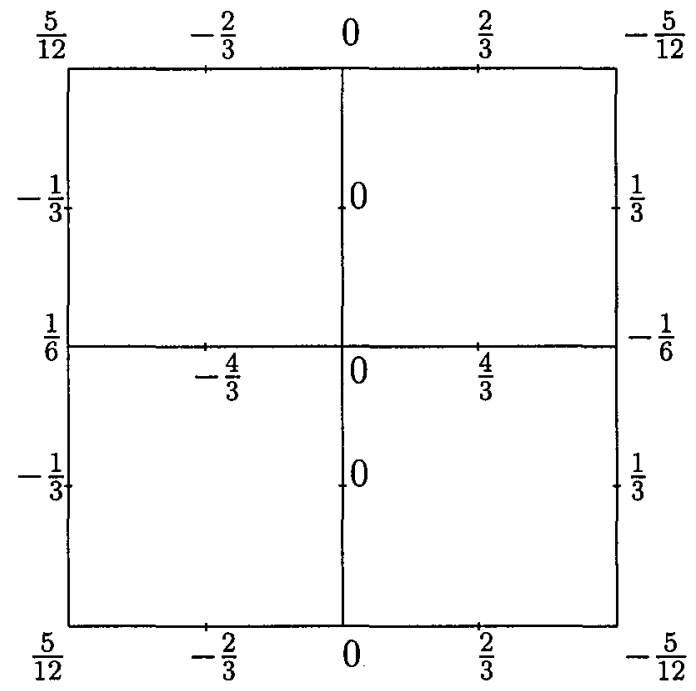

Figure 1: Recovery operator weights $\vec{c}_{j}$ at a vertex 


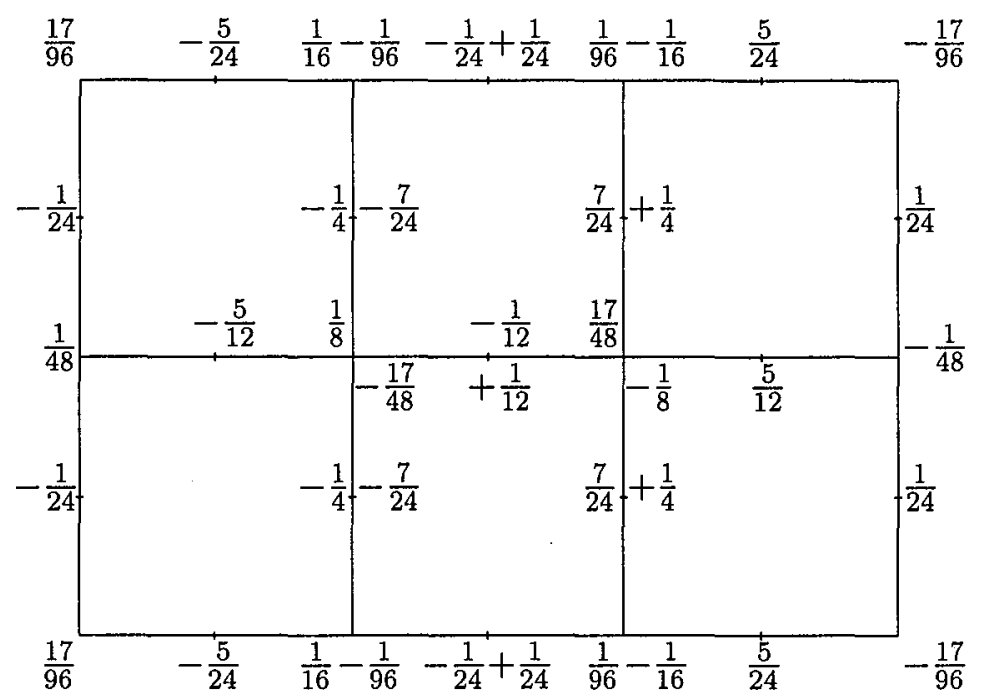

Figure 2: Recovery operator weights $\vec{c}_{j}$ at a horizontal edge center

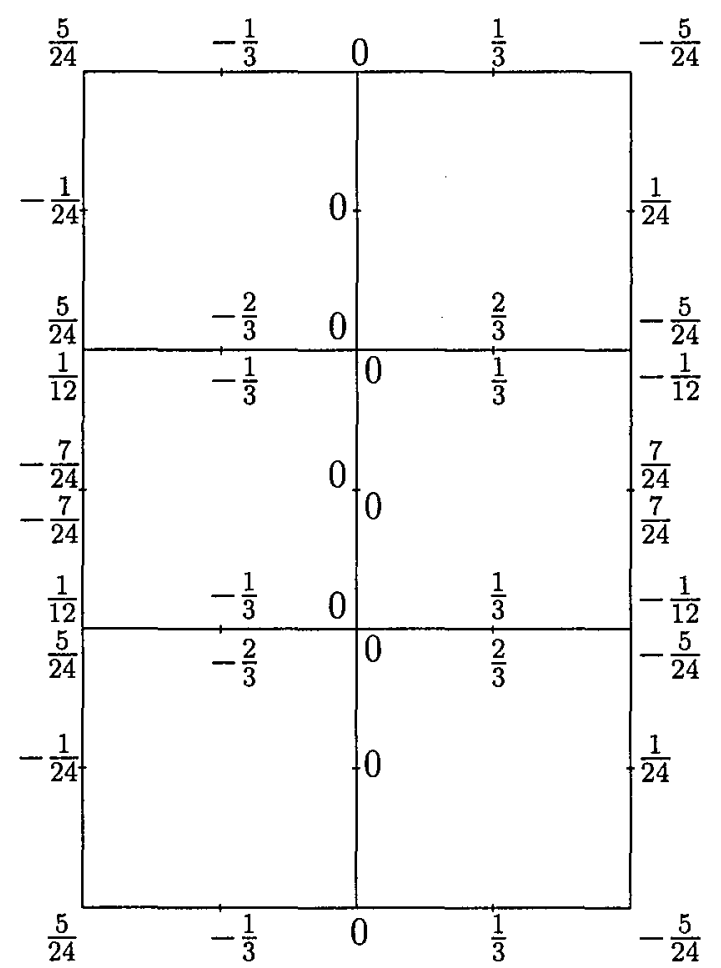

Figure 3: Recovery operator weights $\vec{c}_{j}$ at a vertical edge center 


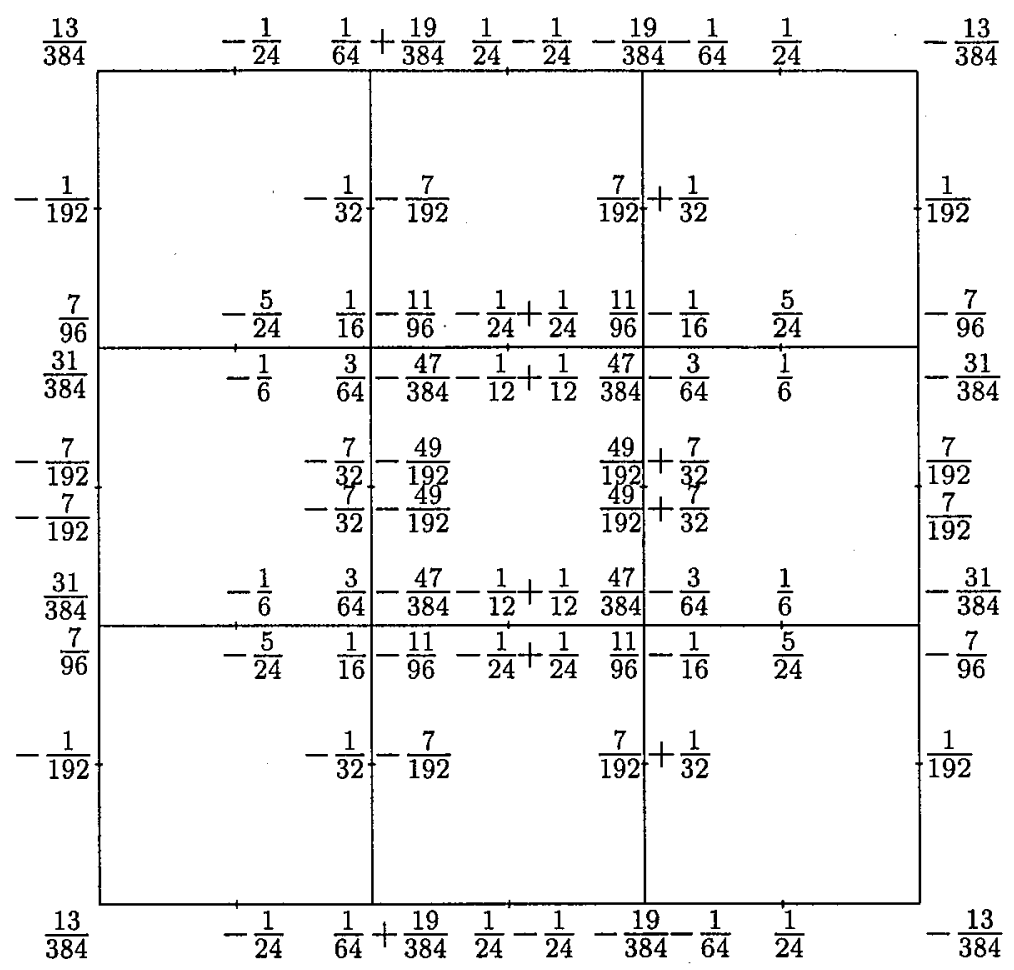

Figure 4: Recovery operator weights $\vec{c}_{j}$ at an element center

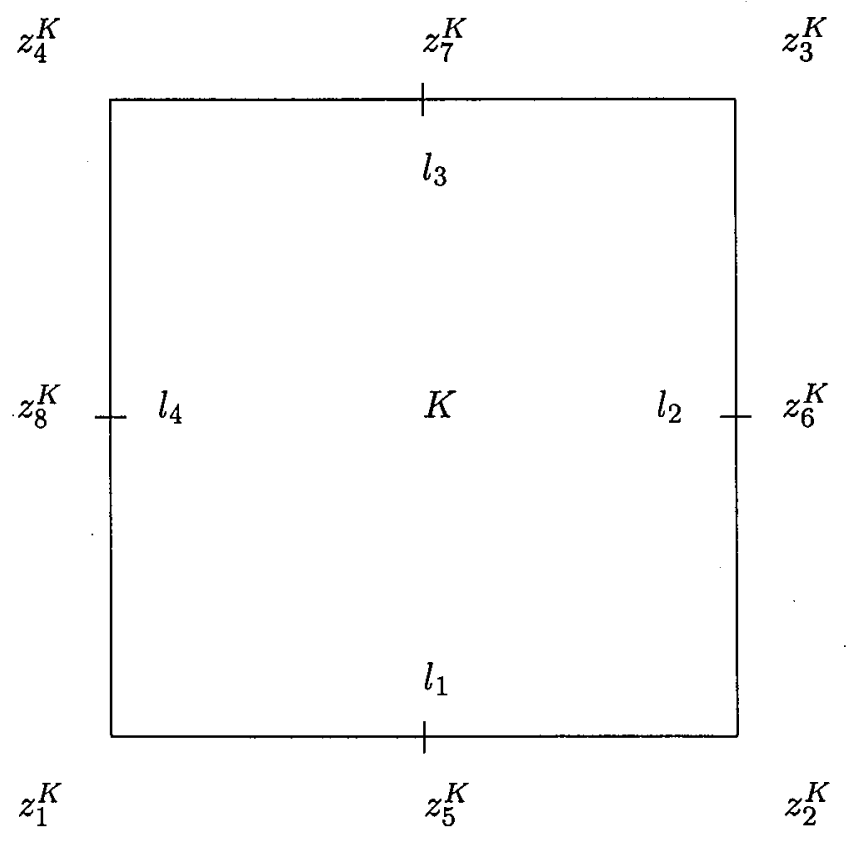

Figure 5: Q8 Element 


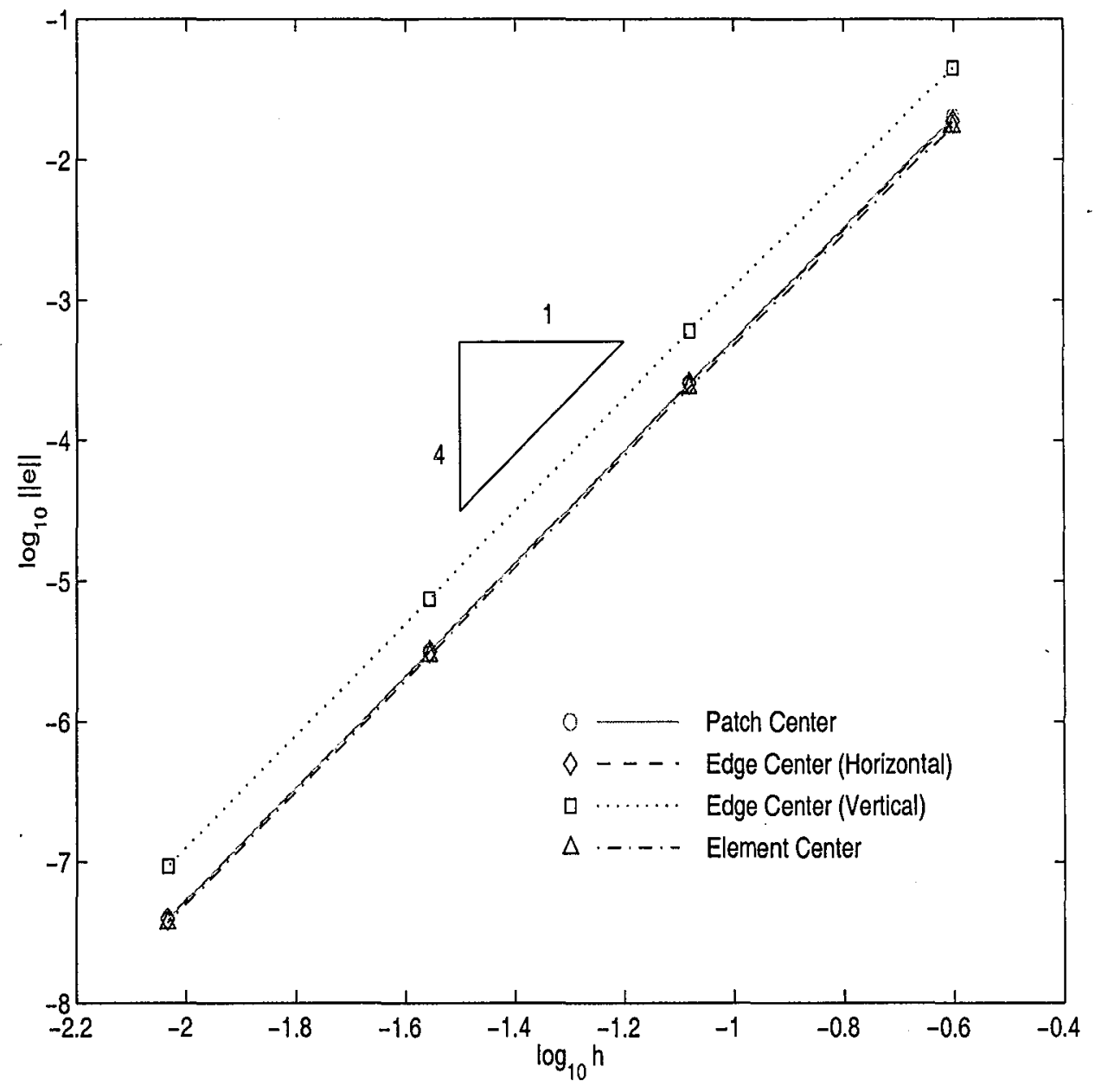

Figure 6: Convergence rate for recovered $x$-derivative: Example 1 


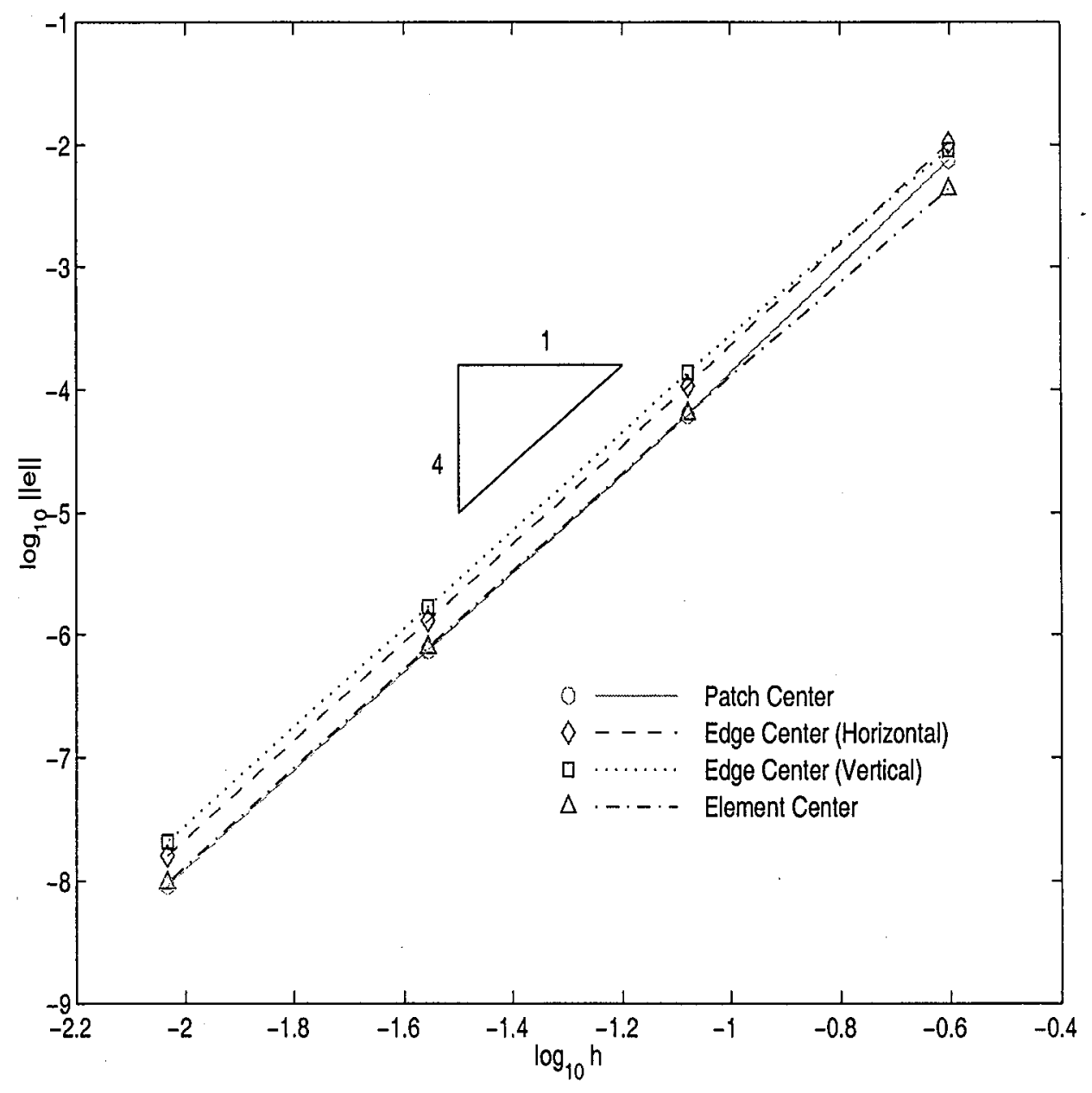

Figure 7: Convergence rate for recovered $x$-derivative: Example 2 


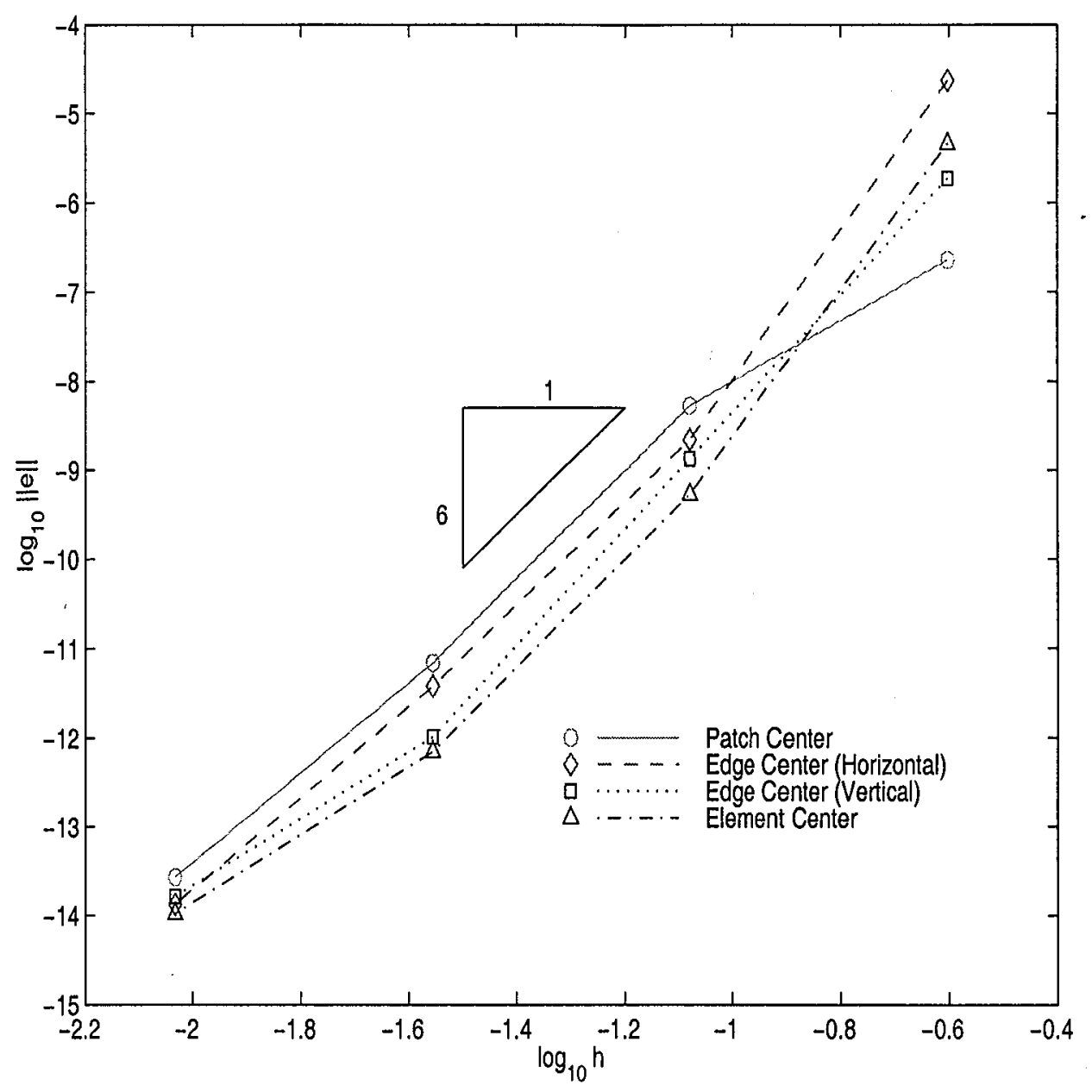

Figure 8: Convergence rate for recovered $x$-derivative: Example 3 